\title{
Retrieval of reflections from ambient noise recorded in the Mizil area, Romania
}

\author{
Ionelia Panea ${ }^{1}$, Deyan Draganov², Carlos Almagro Vidal ${ }^{2}$, and Victor Mocanu ${ }^{1}$
}

\begin{abstract}
We applied seismic interferometry (SI) by crosscorrelation to ambient-noise panels recorded in the Mizil area, Romania, aiming to retrieve body-wave reflections. To achieve this goal, surface waves in the noise panels input to SI should be suppressed. We did this by selecting for input to SI-only noise panels that are not dominated by surface waves; the selection was either after visual inspection in the time domain or after automatic slowness evaluation on crosscorrelated panels. The latter used the slowness of arrivals passing through the virtual-source position at time $0 \mathrm{~s}$. We discovered that the automatic slowness-evaluation method allows better retrieval of reflections. From the retrieved reflection gathers, we obtained stacked images of the subsurface. Comparing the SI images to a stacked image from active-source data, we concluded that some retrieved events correspond to reflectors in the active seismic section, including known geologic markers. In a previous application of SI to ambient noise, the retrieved reflections exhibited frequency content lower than that of active data. In our results, the frequency content of the SI retrieved data was comparable to the one of the active data.
\end{abstract}

\section{INTRODUCTION}

Seismic interferometry (SI) is known as a method that can retrieve the Green's function between two stations from crosscorrelation of recorded responses at these stations from surrounding sources. In the following sections, we show results from application of this method to ambient seismic noise for retrieval of reflections. The SI method is based on the acoustic daylight-imaging principle proposed by Claerbout (1968) for a 1D medium and which auto- correlates the transmission response from a deep noise source. Later, the 1D method was extended to a general 3D medium using reciprocity theorems of the correlation and convolution type (Wapenaar et al., 2002; van Manen et al., 2005; Wapenaar and Fokkema, 2006) and using stationary-phase analysis (Snieder, 2004). This resulted in the development of a robust theory that describes the retrieval of the full 3D Green's function from crosscorrelations, meaning the retrieval of surface and body waves (including reflections). SI by crosscorrelation can be applied to naturally occurring transient or noise sources, but also to active man-made sources (Schuster, 2001; Bakulin and Calvert, 2004; Schuster et al., 2004). Even though the theory was developed for a lossless medium surrounded by a boundary of sources, Snieder (2007) shows that Green's function can still be retrieved if the receivers are surrounded by a complete volume of sources and the source density is proportional to the intrinsic losses. Having a complete volume of sources would be difficult to achieve in the field. Because of this, alternatives have been proposed. Instead of using crosscorrelation, SI can be applied using crossconvolution (Slob et al., 2007; Halliday and Curtis, 2009), but this requires that one of the receivers be outside of the enclosing boundary sources. Another alternative is to use trace deconvolution (Vasconcelos and Snieder, 2008a, 2008b). The SI by trace deconvolution is based on an idea proposed by Riley and Claerbout (1976). Bitri et al. (2011) compared the SI responses obtained using crosscorrelation and trace deconvolution on surfacewave signals generated by controlled-noise sources. They showed that reliable dispersion curves of the surface waves could be obtained by both methods, but that SI responses obtained by trace deconvolution are better, increasing the accuracy of the S-wave velocity distribution derived from the analysis of the surface waves. SI can also be applied by multidimensional deconvolution (Wapenaar et al., 2008). The advantage of using multidimensional deconvolution is that in addition to taking intrinsic losses into account, it can also compensate for inhomogeneous illumination from the sources (Wapenaar et al., 2011a, 2011b).

\footnotetext{
Manuscript received by the Editor 2 August 2013; revised manuscript received 29 December 2013; published online 9 May 2014

${ }^{1}$ University of Bucharest, Faculty of Geology and Geophysics, Bucharest, Romania. E-mail: ipanea2@yahoo.com; vi_mo@yahoo.com.

${ }^{2}$ Delft University of Technology, Department of Applied Geophysics and Petrophysics, Delft, The Netherlands. E-mail: d.s.draganov@tudelft.nl; c.almagrovidal@tudelft.nl.

(C) 2014 Society of Exploration Geophysicists. All rights reserved.
} 
These SI methods retrieve the Green's function between two receivers. But the SI process can also be applied to retrieve the Green's function between two sources (Curtis et al., 2009) or between a source and receiver, where the receiver has not recorded the signal from that source (so-called source-receiver interferometry, Curtis and Halliday, 2010).

Even though SI by crosscorrelation for retrieval of Green's function between two receivers has its shortcomings, it is a very stable and easy-to-apply method. That is why it has gained popularity quickly, including for retrieval of surface waves from ambient noise generated by sources located near or at the earth's surface (e.g., Shapiro and Campillo, 2004; Sabra et al., 2005). The retrieved surface waves can be further used as input data for regional tomographic imaging (Shapiro et al., 2005; Yao et al., 2006) or monitoring changes in volcanic chambers (Sens-Schönfelder and Wegler, 2006; Brenguier et al., 2008).

SI by crosscorrelation can also be used to retrieve body-wave arrivals from passive seismic. This has been done using teleseismic arrivals (Ravi Kumar and Bostock, 2006; Tonegawa et al., 2009; Ruigrok et al., 2011), but also using ambient noise. Roux et al. (2005) succeed in retrieving diving P-waves in such a way. Crosscorrelation of recorded ambient noise has also resulted in the retrieval of body-wave reflections (Hohl and Mateeva, 2006; Draganov et al., 2007; Draganov et al., 2009; Zhan et al., 2010; Ruigrok et al., 2011; Xu et al., 2012). Note that retrieval of body waves by SI with ambient noise has proved more difficult, as the body-wave noise is normally drowned out by the much stronger surface-wave noise. To enhance retrieved body-wave arrivals, the surface waves should be suppressed. This can be done by choosing a specific frequency band, where the body waves are dominant (Ruigrok et al., 2011); by choosing specific intrastation distances, e.g., close to the critical angle for the S-wave reflections (Zhan et al., 2010); and by groups of receivers (Draganov et al., 2009).

If the reflection response of the subsurface is retrieved from ambient noise or teleseismic arrivals for different positions of the virtual sources and the receivers, one can proceed to process the

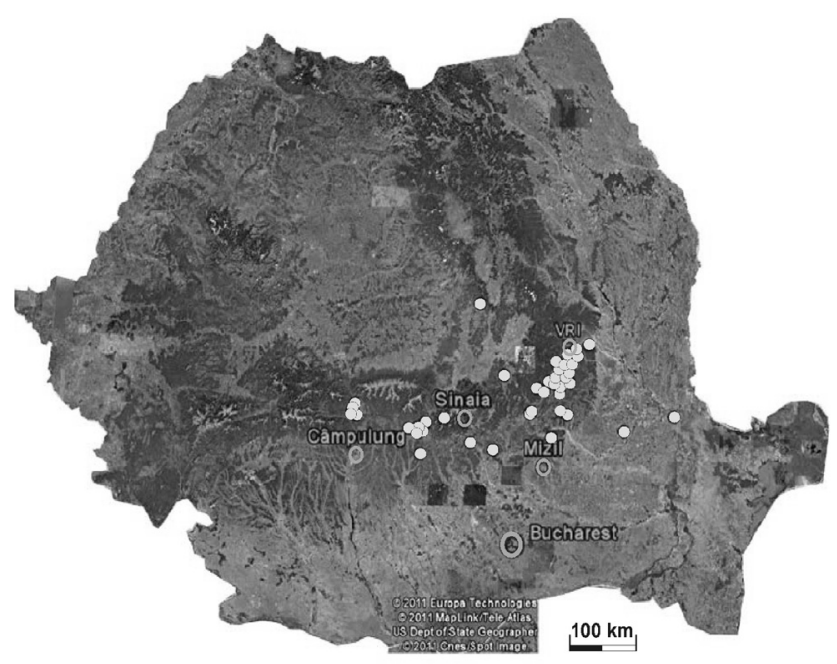

Figure 1. Map of Romania indicating the active seismic Campulung-Sinaia-Vrancea area and the position of the town of Mizil. The light gray dots show the epicentra of the earthquakes, which were registered during April 2009. Source of the map: http:// maps.google.com. reflection data to extract information about the geologic structure in the investigated area. This can be achieved using stacking and migration techniques as commonly used in the exploration seismology. The reflected and refracted energy in the correlated panels and virtual common-source gathers can be analyzed to determine the velocity distribution into the subsurface (King and Curtis, 2011).

In the following, we use SI by crosscorrelation to retrieve reflected waves from ambient-noise recordings. As mentioned above, the retrieved reflected waves might be weaker than retrieved surface waves. Draganov et al. (2010a) show that crosscorrelating only those parts of the ambient noise, which contain identifiable body-wave noise, results in stronger retrieved reflections. We follow this approach and inspect the noise to separate it into surface-wave and body-wave dominated parts. We then use the latter part to retrieve reflection arrivals and proceed to obtain stacked sections of the subsurface.

\section{DESCRIPTION OF THE PASSIVE SEISMIC SURVEY}

The passive seismic survey was performed in the Mizil area, Romania, which is close to the seismically active Campulung-SinaiaVrancea zone (Figure 1). Two types of earthquakes occur in this area, namely shallow earthquakes, with hypocenters located between 20 and $60 \mathrm{~km}$ depth and magnitude lower than 5.6, and intermediate earthquakes, with hypocenters located between 60 and $200 \mathrm{~km}$ depth and magnitude up to 7.4 or even higher (Oncescu and Bonjer, 1997). The continuous seismic activity present in this area provides significant amounts of data that contain information about the seismic reflectivity and the elastic parameters of the subsurface and makes it very attractive for an SI experiment for retrieval of reflected body waves from ambient noise. The passive measurements were taken on 24 April 2009. During April 2009, numerous earthquakes occurred and we indicate their epicenters in Figure 1 by the light gray dots.

Another reason for performing the passive measurements in this area was the availability of active-source seismic reflection data with multiple sources recorded along a profile, which crossed the town of Mizil (Figure 2). These data are used for comparison with the SI results.

The ambient-noise acquisition was done over a period of $6 \mathrm{~h}$ using two Geodes seismic recorders (Geometrics) with 24 verticalcomponent geophones each; the natural frequency of the geophones was $4.5 \mathrm{~Hz}$. The geophones were planted along two orthogonal directions (northeast and northwest), where one of the directions was parallel to the active-source seismic reflection lines (Figure 2). The geophones were spaced at $2.5 \mathrm{~m}$ and were labeled with field numbers G1 to G24 on both lines starting from the eastern-most and the northern-most positions on the northeast and northwest lines, respectively. The time-sampling interval was $1 \mathrm{~ms}$, allowing the recording of arrivals with frequencies up to $500 \mathrm{~Hz}$. Autosaving of the recorded ambient noise was done after each second. The recorded seismic energy was due to natural (possibly weak earthquakes) and anthropogenic sources (e.g., passing cars, trains, etc.). Due to the field conditions, the geophone lines were placed approximately $1 \mathrm{~km}$ away from a railway, which represented a source of occasional strong coherent noise (surface waves) seen on the records. Some passive records contain surface-wave noise generated by cars passing infrequently along a secondary road situated in the vicinity of the geophone lines (Figure 2). Quality control of the recorded noise 
was performed in the field to verify that the measurements were performed properly.

\section{ANALYSIS OF THE PASSIVE DATA}

The passive seismic data are represented by 1183 ambient-noise panels of $16 \mathrm{~s}$ length. To obtain these, the $1 \mathrm{~s}$ noise panels recorded in the field were vertically concatenated to $16 \mathrm{~s}$ panels for easier further handling.

As a first step in the analysis of the noise, we look at the spectrum of the noise for possible identification of bands where the bodywave noise might not be completely covered by surface-wave arrivals. Figure 3 shows the spectra up to $60 \mathrm{~Hz}$ for the (a) northeast and (b) northwest line. The spectra were obtained by summation of all the traces in a noise panel and Fourier transformation. This is repeated for all noise panels, and the separate spectra are summed together. By summation of the traces in a noise panel before Fourier transform, we aim to suppress the random (noncoherent along the traces) part of the recorded noise (e.g., instrument noise) and to suppress surface waves, but to preserve the possible body-wave arrivals in the noise, which are almost horizontal in each panel due to its limited offset range. SI uses the latter to retrieve the arrivals between the seismic stations. For the northeast line, we can see strong amplitudes up to $11 \mathrm{~Hz}$. From previous experiments with noise data, but also from the active data in the area, we attribute these to anthropogenically generated surface-wave noise. Note that along the northwest line the energy up to $10 \mathrm{~Hz}$ is not that strong, even though it again dominates the spectrum. Due to the presence of this energy, we choose to look at the noise above $11 \mathrm{~Hz}$. Above $11 \mathrm{~Hz}$ and up to approximately $25 \mathrm{~Hz}$, the amplitude spectrum gradually weakens and around $28 \mathrm{~Hz}$ is at its lowest level. Above that frequency, the amplitude slowly increases again up to the peak at $50 \mathrm{~Hz}$, the latter caused by the power lines in the area. Looking at the am-

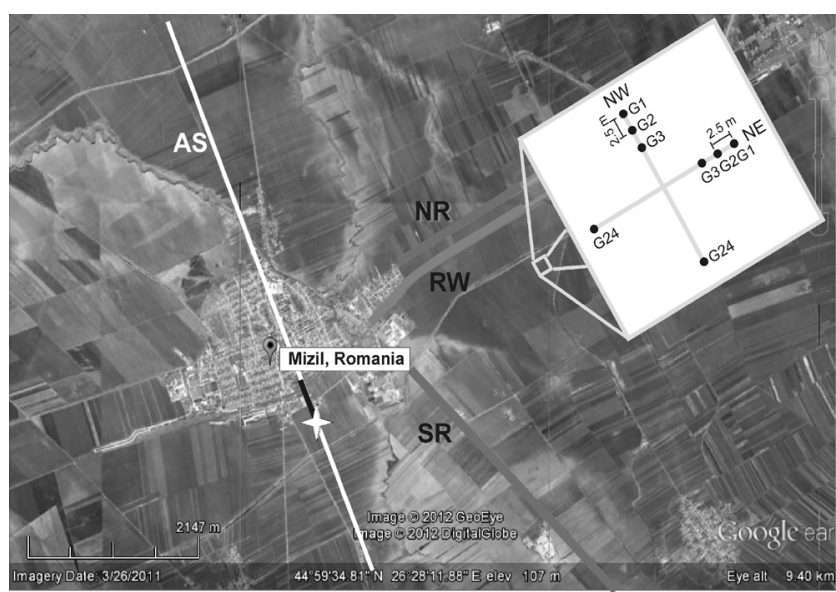

Figure 2. Map of the Mizil area showing the position of the passive experiment (the gray rectangle) (AS, active seismic reflection line (the thick white line); NR, national road; SR, secondary road; RW, railway line - all three are indicated also by the thick gray lines. The white star indicates the location of the source for the active shot record whose spectrum is shown in Figure 3c. The inset shows the geometry of the passive array, consisting of two orthogonal lines of 24 vertical geophones (labeled with field numbers G1 to G24) spaced at $2.5 \mathrm{~m}$. The black part on the AS indicates the projection of the coordinates of the northwest passive line. Source of the map: http://maps.google.com. plitude spectrum of a shot gather recorded along the active profile AS approximately $3 \mathrm{~km}$ from the passive survey, we notice strong amplitudes between 15 and $22 \mathrm{~Hz}$ (Figure 3c). This is likely caused by the used sources, but also by the intrinsic losses in the subsurface. Nevertheless, seeing that the active data have their main energy in this frequency interval and that the passive data also have possibly body-wave noise in the same interval is encouraging because it means that if we succeed to retrieve reflections from the ambient noise, they would be comparable in frequency content to the active data. Because of all these features, we choose to concentrate our search for body-wave noise in the frequency band between 11 and $23 \mathrm{~Hz}$. The higher limit is dictated by the lack of energy above $30 \mathrm{~Hz}$; the apparent increase in the amplitudes in the spectra is caused by the build-up of the peak at $50 \mathrm{~Hz}$ and by the presence of a weaker peak in the spectra around $25 \mathrm{~Hz}$ (resulting from the $50-\mathrm{Hz}$ peak), which we want to avoid. Note that in the interval from 11 to $23 \mathrm{~Hz}$, the amplitude spectra of the active and the passive data are quite similar.

We display in Figure 4 examples of ambient-noise panels (filtered between 11 and $23 \mathrm{~Hz}$ ). The panels in Figure 4a-4d are clearly dominated by surface-wave noise because at least along one of the perpendicular lines, the events exhibit linear moveout with relatively low apparent velocity. The panels in Figure $4 \mathrm{e}$ and $4 \mathrm{f}$ show arrivals that have very high apparent velocities along both perpendicular lines and thus are not surface waves. Mizil is a small town with a population of approximately 16,000 inhabitants with agriculture as the main activity and very little industry. The traffic to and from the town, even during the day hours when the passive measurements were performed, was low. Still, the roads and railway acted as sources of strong surface waves even in the chosen frequency band (see Figure $4 \mathrm{a}-4 \mathrm{~d}$ for examples). For the case of the noise panels displayed in Figure $4 \mathrm{a}-4 \mathrm{~d}$, we obtained values for the surface-wave velocities ranging between 150 and $290 \mathrm{~m} / \mathrm{s}$. The same surfacewave arrivals exhibit linear and hyperbolic moveout on the two orthogonal lines due to the position of the noise sources with respect to each of the geophone lines. Nevertheless, we can see in Figure $4 \mathrm{e}$ and $4 \mathrm{f}$ that in the chosen frequency band some of the noise panels are not dominated by surface waves. The horizontal arrivals on both panels actually represent body-wave noise.

The ambient-noise panels are used as input data for SI by crosscorrelation. Depending on the type of wave, we want to retrieve,

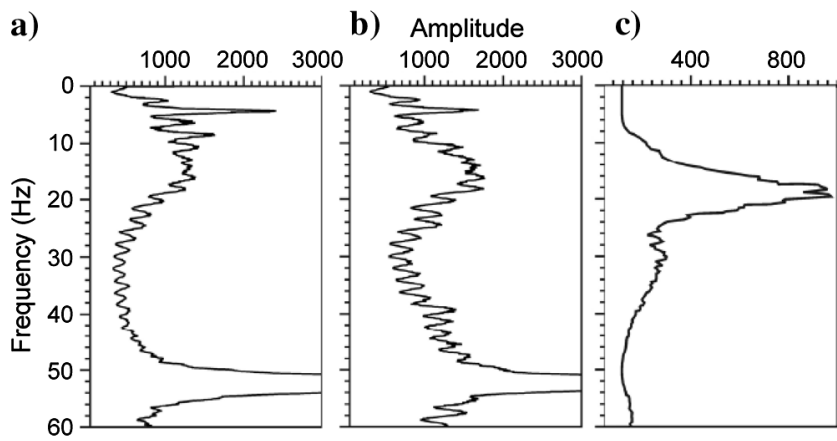

Figure 3. Summed frequency spectra of the Fourier transformed individual recorded noise panels along the (a) northeast and (b) northwest line. The traces in each noise panel were summed before Fourier transformation. (c) Frequency spectrum of the active shot gather recorded in the vicinity of the passive survey. 
surface or reflected wave the ambient-noise panels can be considered suitable or not suitable for retrieval (Draganov et al., 2010a, 2013). For example, if we want to retrieve surface waves, the noise panels displayed in Figure $4 \mathrm{a}-4 \mathrm{~d}$ are suitable as an input to SI, but if we want to retrieve reflected waves, then the same noise panels are not suitable because they are dominated by surface waves and after crosscorrelation retrieved surface waves will dominate the SI result. Any possible reflection information would be buried under the retrieved surface waves. On the other hand, the ambient-noise panels in Figure $4 \mathrm{e}$ and $4 \mathrm{f}$ are suitable for retrieval of reflections because they contain body-wave noise. Figure $5 \mathrm{a}$ and $5 \mathrm{~b}$ shows the frequency-wavenumber spectrum of the unfiltered surface-wave dominated noise panels from Figure $4 \mathrm{a}$ and $4 \mathrm{~b}$, respectively. Note that we do not apply here frequency band-pass filtering to show that surface-wave energy, characterized by the inclined lines, is present along the complete frequency spectrum. The $2.5 \mathrm{~m}$ receiver spacing implies that surface-wave noise above approximately $25 \mathrm{~Hz}$ is spatially aliased. Therefore, using a high-cut filter at $23 \mathrm{~Hz}$ avoids the risk of selecting surface-wave energy that is spatially aliased.

\section{RESULTS FROM THE APPLICATION OF SEISMIC INTERFEROMETRY}

The equation used to compute the SI responses is

$$
\begin{gathered}
\left\{G_{z, z}\left(x_{A}, x_{B}, t\right)+G_{z, z}\left(x_{A}, x_{B},-t\right)\right\} * s(t) \\
\approx \sum_{i=1}^{N} v_{z}^{\mathrm{obs}, i}\left(x_{A}, t\right) * v_{z}^{\mathrm{obs}, i}\left(x_{B},-t\right),
\end{gathered}
$$

where $G_{z, z}\left(x_{A}, x_{B}, \pm t\right)$ denote Green's function and its time-reversed version measured in the vertical direction at $x_{A}$ due to a vertical source at $x_{B}, t$ denotes time, $v_{z}^{\text {obs, } i}$ represents the component of the particle velocity recorded in the vertical direction in the $i$ th noise panel, $s(t)$ stands for the averaged autocorrelation of the source time function of the noise sources, $N$ is the number of noise panels used, and the asterisk denotes convolution. We have adapted equation 1 from Wapenaar and Fokkema (2006), by exchanging the ensemble average in the original relation with summation over the available noise panels. a)

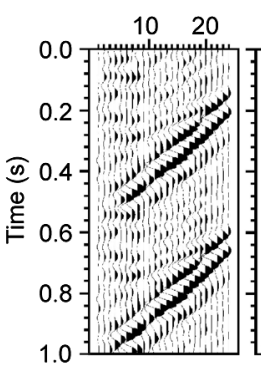

b)

c)

d) Geophone number
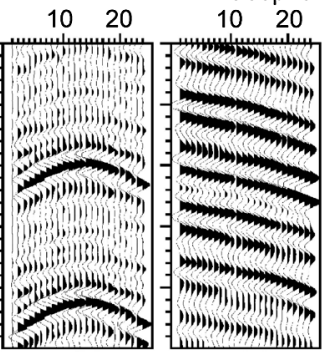

e)

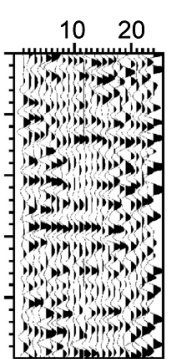

Figure 4. Recorded ambient-noise panels filtered between 11 and $23 \mathrm{~Hz}$ : (a and b) with surface waves from a passing car along the secondary road; (c and d) with surface waves from a passing train; and (e and f) without interpretable surface waves, but possibly with body-wave noise. The panels in (a, c, and e) are recorded along the northeast geophone line, and in panels (b, d, and f) are recorded along the northwest line; see the inset in Figure 2.

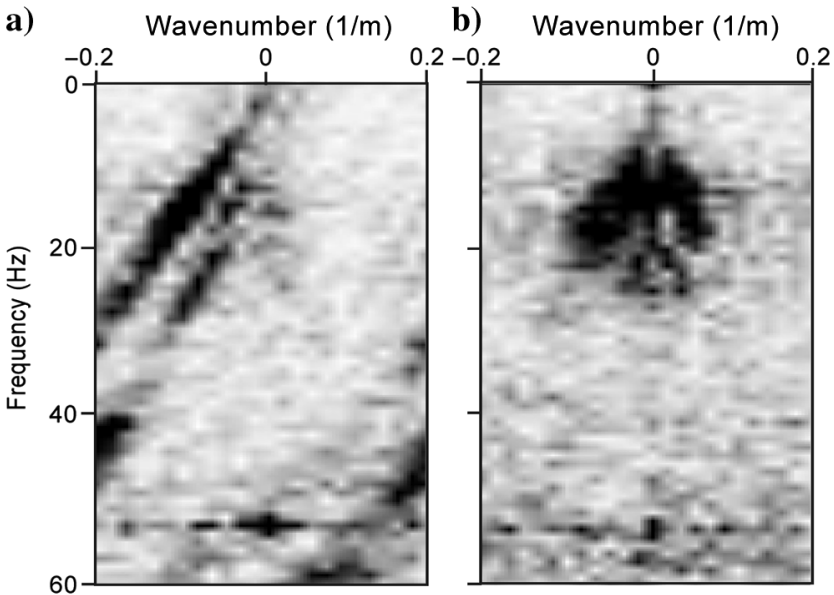

Figure 5. Frequency-wavenumber amplitude spectra of the noise panels with dominant surface waves displayed in (a) Figure 4a and (b) Figure 4b. Frequency band-pass filtering is not applied to show the aliasing of the surface waves at higher frequencies. f)

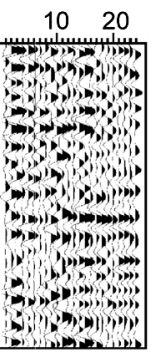

\section{Retrieval of surface waves from ambient noise}

As discussed by Draganov et al. (2010a, 2013), correlation of noise panels characterized by dominant surface-wave noise would mainly retrieve surface waves, whereas retrieval of reflections would result from correlation of noise panels with dominant or at least identifiable body-wave noise. The same authors also show that by inspecting visually the recorded noise and separating it into surface-wave dominated and body-wave dominated, they could improve the signal-to-noise ratio of the retrieved reflection arrivals. We also follow this procedure for separation of the passive records in noise panels with and without dominant surface waves. As a first step, we do this using visual inspection by displaying the noise records in the time domain after band-pass filtering between 11 and $23 \mathrm{~Hz}$. A short length of the recorded noise (one noise panel of $16 \mathrm{~s}$ ) is visualized simultaneously along the northeast and northwest lines. If the noise is not dominated by surface waves on both lines, then this noise panel is input for further retrieval of reflections; otherwise, it is input for further retrieval of surface waves. As a result, we obtain two subsets - one with dominant surface waves, of approximately $2 \mathrm{~h}$ length in total, and a second without dominant surface waves of approximately $4 \mathrm{~h}$ total length (786 noise panels). A disadvantage of splitting the data is that we are left with a smaller number of noise panels for the application of SI for each wave mode. This might lead to weaker body-wave arrivals being missed because they are below the correlation-noise level.

We apply equation 1 to the surface-wave-dominated data set in the following way. First, all $16 \mathrm{~s}$ noise panels are energy normalized. Then, a master trace is chosen - this is the trace at the location of which we will obtain a virtual source $\left(x_{B}\right.$ in equation 1$)$. The master trace from one noise panel is correlated with all the traces of the same panel (multiple traces $x_{A}$ in equation 1) to obtain a correlated panel (the quantity inside the summation sign in equa- 
tion 1). This is repeated for all selected noise panels, and the resulting correlated panels are summed together. Each noise panel contains 24 traces, which is the maximum number of geophones on each of the passive lines. The SI responses are obtained following the described-above correlation and summation operation resulting in 24 summed correlated panels (24 virtual commonsource gathers with retrieved results at positive and negative times).

To obtain the final virtual common-source gathers, summation of the causal and acausal times of the summed correlated panels could be done. Theory states that if the noise sources illuminate the passive array from all directions with equal strength (homogeneous illumination), the causal and acausal parts will be retrieved and will be equal. The SI response can then be obtained by taking either of the two parts. When the illumination is not homogeneous, some parts of Green's function might be retrieved only at causal times and some parts only at acausal times, meaning that more complete virtual-source gathers might be obtained by summing the timereversed acausal part with the causal part. In our study, the latter is also the case, especially for the surface-wave noise. There are two dominant illumination directions for surface-wave energy, namely, the secondary road and the railway (Figure 2). Due to the short lengths of the lines and relatively simple subsurface geology, known from the active exploration survey, instead of summing the complete causal and time-reversed acausal parts, we apply the approach from Ruigrok et al. (2011) and use (concatenated) parts of the positive and parts of the negative times. Assuming a (nearly) horizontally layered subsurface, a plane wave incident with a dip in the direction of the increasing geophone numbers after application of SI will result in a retrieval of a plane wave as well with the same dip. The retrieved plane wave will be passing through the trace of the virtual source at $0 \mathrm{~s}$. Due to the preserved dip, the retrieved plane wave will be present only at negative times for geophone number smaller than the number of the virtual-source geophone and only at positive times for geophones with number bigger than the number of the virtual-source geophone. If the negative-time part of the retrieved plane wave is taken and flipped in time, it would be identical (in a traveltime sense) to a retrieved plane wave with a dip opposite to the original incident plane wave. A plane wave with such opposite dip would normally be retrieved at positive times by the application of SI to an incident plane wave with a dip in the direction of the decreasing geophone numbers. This means that use of the method of Ruigrok et al. (2011), i.e., of using the positive times to one side of the virtual source and of the flipped-intime negative times to the other side of the virtual source, would effectively result in balanced illumination from both sides of the array. In our case, for the northeast line, the main surface-wave illumination is from the secondary road (the railroad-related noise is present on few panels). Because of this, for geophones with a station number greater than the number of the mastertrace geophone, we use the retrieved time-reversed acausal times, while for geophones with a lower station number, we use the causal times.

The virtual common-source gathers obtained for the master trace at geophones G1, G6,
G12, G18, and G24 are displayed in Figure 6. We observe clear surface waves characterized by high amplitudes and similar velocities. Note that the retrieved surface-wave arrivals cover the complete noise panel. This is due to the spatial and temporal correlation of the main sources of surface-wave noise. No other retrieved arrivals can be distinguished, which confirms that when noise dominated by surface wave is crosscorrelated, only surface waves would be retrieved, even if body-wave noise is present.

As mentioned above, rejecting noise panels might result in weaker reflections not being retrieved above the correlation-noise level. Because the total number of the noise panels with dominant surface waves amounts to length approximately one-third of the total length of the recorded noise, it would be better to suppress the surface-wave noise and use also these panels for retrieval of reflections. We use array forming to attempt to attenuate the surface waves. The responses of two types of arrays were computed: arrays with five and 12 elements. Having single-sensor records, the array response can be computed in two steps (Hoffe et al., 2002; Panea and Drijkoningen, 2008). First, we sum as many traces as the desired number of array elements and then we resample the output at the desired group interval. The group interval is the distance between two consecutive arrays. For our data, due to the small number of geophones used in the field for data acquisition, the group interval is equal to the geophone spacing to output as many traces as possible. The array with five elements suppresses arrivals with wavenumbers between $0.08 \mathrm{~m}^{-1}$ and $0.32 \mathrm{~m}^{-1}$, whereas the array with 12 elements suppresses arrivals with wavenumbers between $0.033 \mathrm{~m}^{-1}$ and $0.366 \mathrm{~m}^{-1}$.

In Figure 7, we display the results of the array-forming with five and 12 elements in the time-space and frequency-wavenumber domains for an unfiltered noise panel recorded along the northeast line. The wavenumber transform was along the northeast line as well. Comparing the results of the array forming, we see that the suppression of the surface waves is more successful after array forming with 12 elements. This suppression is clear on the fre-

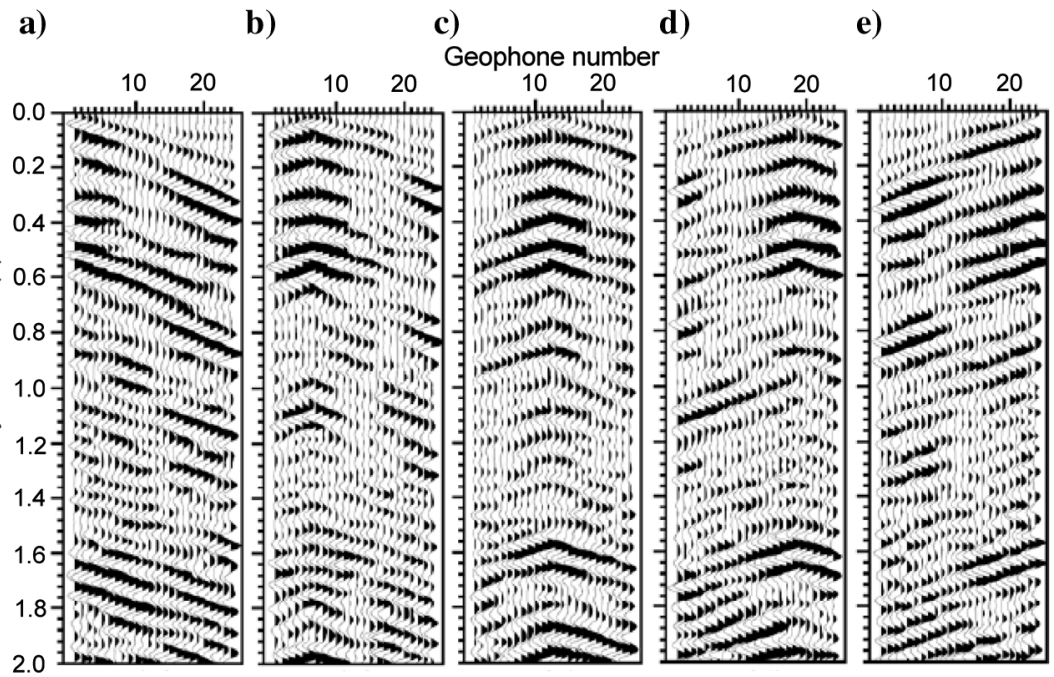

Figure 6. Retrieved common-source gathers along the northeast line from noise panels with dominant surface waves selected after visual inspection of the recorded ambient noise. The virtual source is at geophones (a) G1, (b) G6, (c) G12, (d) G18, and (e) G24 for the northeast line. For visualization purposes, the panels are shown after application of automatic gain control with a time window of $0.5 \mathrm{~s}$. 
quency-wavenumber spectrum of the array response compared to the frequency-wavenumber spectrum of the noise panel from Figure 5a. The remaining surface waves are still strong in amplitude after array-forming with five elements. For this reason, arrayforming with five elements is not sufficient in our case. Even though the array-forming with 12 elements shows promising results, for future use of this technique, one has to bear in mind that it might be dangerous as well. Any surface-wave events arriving from the offline direction, such as the railway-related noise, might be recorded along the northeast line as nearly horizontal arrivals. As the array forming is applied only along the northeast line, such flat arrivals will not be weakened, but on the contrary, they will be amplified. After SI, such events might be misinterpreted as reflections that are brought forward. This means that it would be better to perform such array forming using a $2 \mathrm{D}$ grid if the acquisition geometry allows.

\section{Retrieval of body-wave reflections from ambient noise}

Now, we apply SI to the visually separated data set that contains noise panels without dominant surface waves (in the selected frequency band between 11 and $23 \mathrm{~Hz}$ ). The SI responses were computed using equation 1 for both passive lines. The computation is done in the same way as for the surface waves.

In Figure 8, we display the summed correlated panels for master traces at geophones G1, G6, G12, G18, and G24 for noise recorded along the northeast line. The retrieved causal and acausal times are displayed for clarity only from -1 to $1 \mathrm{~s}$. Figure 8 shows nearly horizontal events, which might be retrieved reflected arrivals. These arrivals are nearly linear with a dominant inclination in the direction of increasing geophone numbers. The hyperbolic shape of any retrieved body-wave reflection would be difficult to observe due to the short line length $(57.5 \mathrm{~m})$. Furthermore, possible preferred illumination of the geophones from certain directions would result in incomplete retrieval of the reflections and possibly contribute to their apparent flatness. The summed crosscorrelation results also exhibit sur- a)
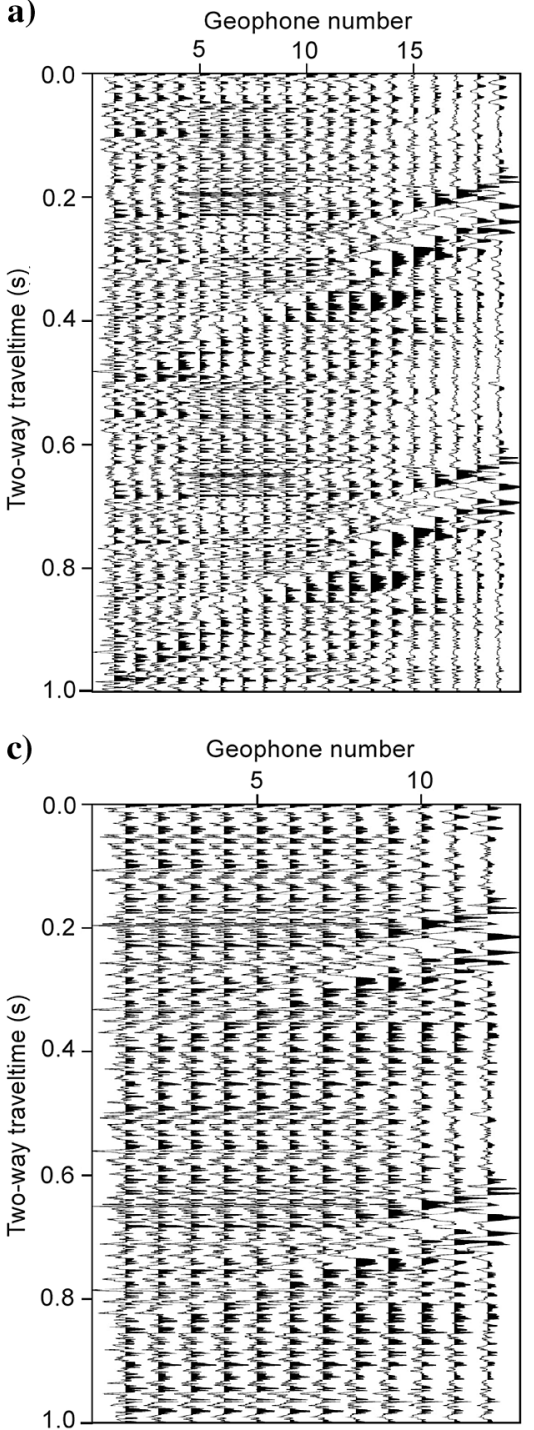

b)

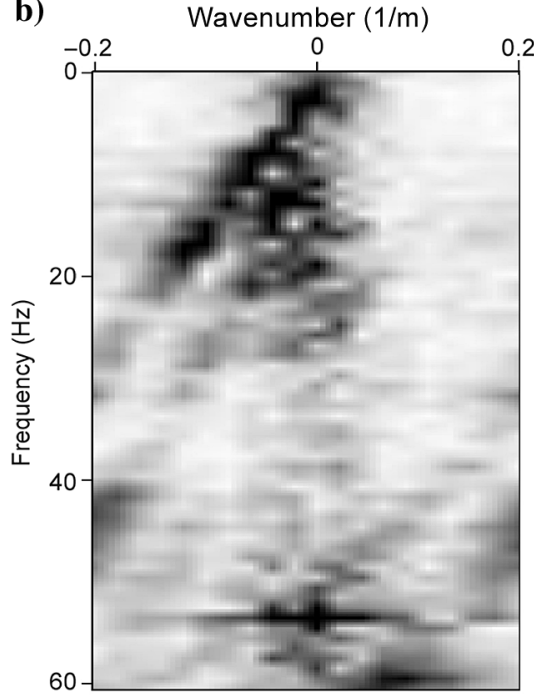

d)

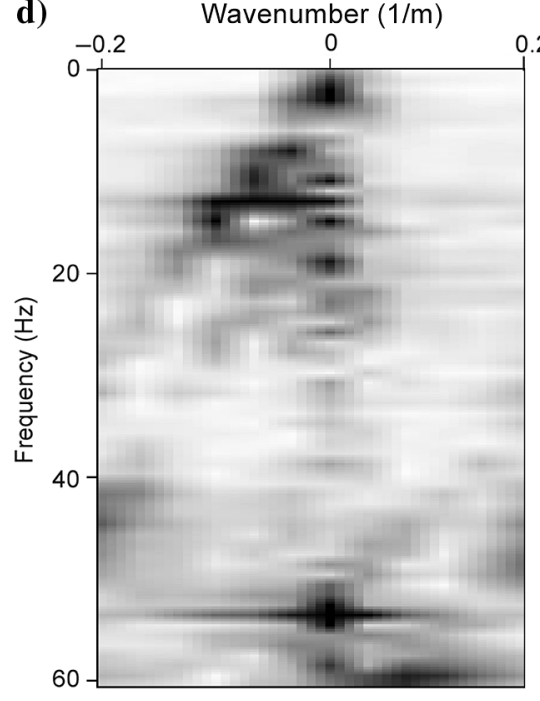

Figure 7. Responses after array-forming with ( $a$ and $b$ ) five elements and (c and d) 12 elements displayed in the (a and c) time and (b and d) frequency domains for a noise panel along the northeast line. face waves, and some of them are characterized by significant amplitude and continuity, for example, the inclined events in Figure $8 \mathrm{~d}$ at geophone numbers $1-12$ and times from $0-0.4 \mathrm{~s}$ and in Figure 8e at geophone numbers 1-20 and times $0-1 \mathrm{~s}$.

The last step in obtaining retrieved virtual common-source gathers is the use of the causal and acausal times of the correlated panels. Due to the present dominant inclination of the retrieved arrivals (but also the short length of the line and the relatively simple subsurface geology), we again apply the approach of Ruigrok et al. (2011): For geophones with a field number smaller than the number of the master-trace geophone, we use the retrieved time-reversed acausal times, whereas for geophones with a higher field number, we use the causal times. These two parts are concatenated to obtain a final retrieved shot gather. In Figure 9, we display the final SI results obtained following this approach. For visualization purposes, the results are displayed after multiplying each time sample by its corresponding time and then applying clipping. The time interval 0-0.6 s exhibits clear possible reflected waves characterized by strong amplitude. Very clear events can be seen also in the time interval $1.5-2$ s. An interesting weak, but continuous event, can be seen at approximately $1.36 \mathrm{~s}$.

Time-variant spectral whitening (not shown) was applied on the retrieved common-source gathers, in addition to the band-pass frequency filtering. The whitening, though, did not result in enhancement of the possible retrieved bodywave reflected waves, possibly due to the spectrum already being quite balanced.

The obtained virtual common-source gathers are further processed using a standard exploration-seismic flow to obtain a stacked seismic section of the subsurface. We used a simple 
processing flow based on that used for the processing of the active seismic data. We resort the retrieved virtual data into common-midpoint (CMP) gathers, perform interactive velocity analysis on the CMP gathers using semblances, apply normal moveout correction, and stack the so-corrected traces in each of the gathers (Yilmaz, 1999). The interactive velocity analysis was done using velocities from active seismic data as guidance. The velocity model was used to compute and apply the normal-moveout correction and for stacking of traces from the CMP gathers. In Figure 10a, we display at the proper horizontal scale the passive section overlaid on the section obtained from the active data. In Figure 2, the active seismic line is indicated by the white long line labeled AS. Due to the fairly horizontal subsurface layering, the distance of approximately $3 \mathrm{~km}$ between the two sections will not influence the interpretation. By comparing both sections, we note that several reflections on the active section can be traced on the passive section. This means that we have imaged several subsurface reflectors using the ambient noise, including main geologic markers present in the sedimentary cover at the two-way traveltimes of 0.2 and $1.9 \mathrm{~s}$. Another marker at $1.6 \mathrm{~s}$ is possibly also imaged, but that is not unambiguously conclusive from the passive section because there is interference at that time from another event, possibly a remnant from the retrieved surface waves.

Even though the results retrieved from the noise are fair, we consider that they can be improved by using a different way of separating the noise panels into parts with and without dominant surface waves. Almagro Vidal et al. (2011a, 2011b) present a method to perform this selection automatically using slowness diagnostics of the dominant energy. The evaluation is performed for each correlated panel. The method uses arrivals in a correlated panel that pass through the virtual-source position at $t=0 \mathrm{~s}$; the collection of these arrivals represent the virtual-source function (van der Neut, 2013). Such arrivals are retrieved from correlation at the master trace and the rest of the traces in a noise panel of the same arrival, in contrast, for example, to a retrieval of reflections, which result from correlation of a primary at the master trace with its free-surface multiple at the other traces. The virtual-source function contains information about the arrivals that have been correlated and are dominant (surface waves or body waves). To extract this information, for each correlated panel, the events in the virtual-source function are summed along slownesses taken to pass through $t=0 \mathrm{~s}$. If the maximum amplitude corresponds to a slowness value located between predefined slowness limits, the correlated panel is kept for further computation of the SI response aimed at retrieval of reflections. If this is not the case, the correlated panel is rejected from further use. dow of $0.5 \mathrm{~s}$.
We applied this method using slowness limits of +0.001 and $-0.001 \mathrm{~s} / \mathrm{m}$. These slownesses correspond to apparent velocities of $1000 \mathrm{~m} / \mathrm{s}$, which is above the maximum inline surface-wave velocity of $290 \mathrm{~m} / \mathrm{s}$. We chose these values so we can also reject dominant surface-wave noise that arrives at some angle to both orthogonal lines (up to approximately $70^{\circ}$ ). The automatic evaluation was done separately for the northeast and northwest lines. In Figure 11a and 11b, we display the results of the slowness evaluation per correlated panel for the northeast and northwest lines, respectively. Each gray horizontal line represents the slowness evaluation for one correlated panel. The cross along each line in- a)

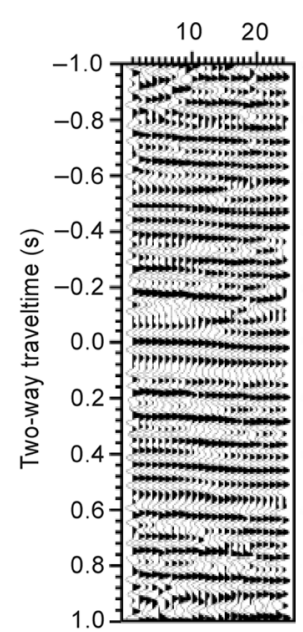

b)

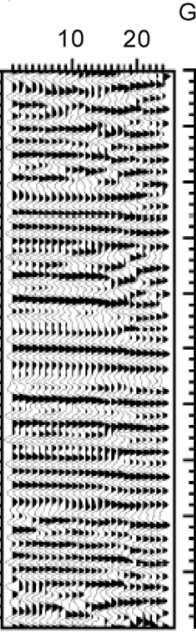

c) Geophone number

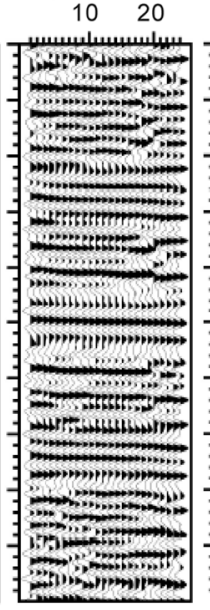

d)

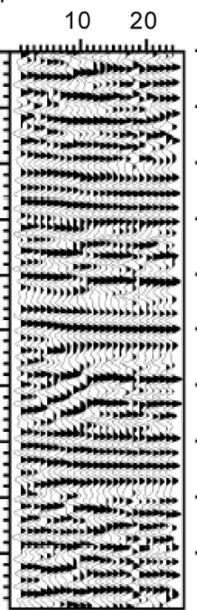

e)

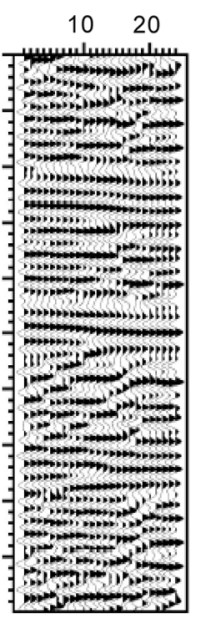

Figure 8. Summed correlated panels obtained from noise panels without dominant surface waves selected after visual inspection of the recorded noise. The master trace is at geophones (a) G1, (b) G6, (c) G12, (d) G18, and (e) G24 along the northeast line. For visualization purposes, automatic gain control has been applied with a sliding time win-

a)

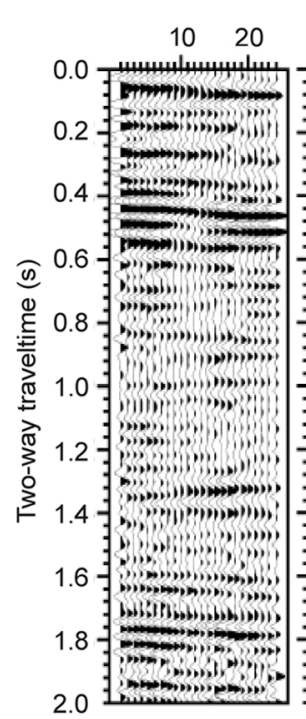

b)

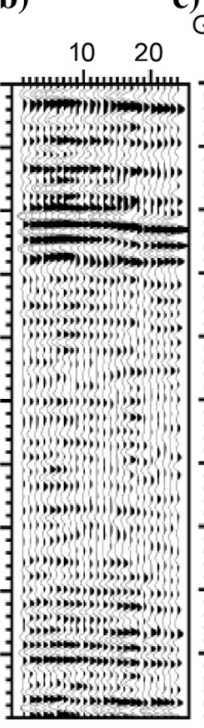

c) Geophone number

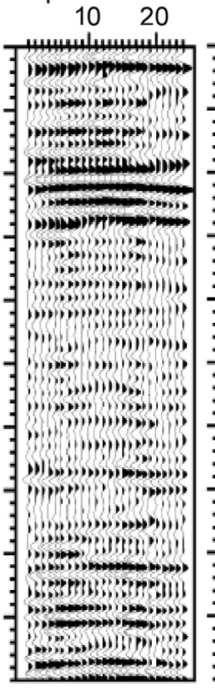

d) $10 \quad 20$

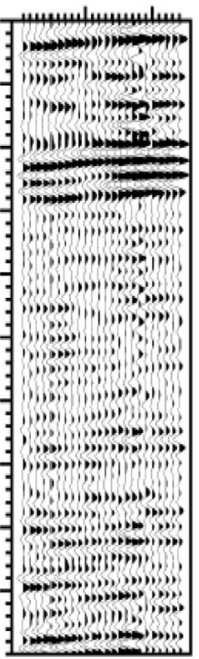

e)

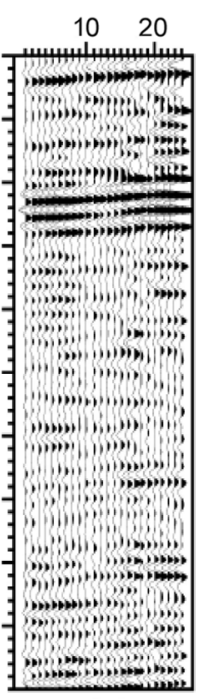

Figure 9. Retrieved common-source gathers from the corresponding panels in Figure 8 after summing parts of the causal and time-reversed acausal times. 
dicates the peak slowness for this correlated panel. A correlated panel is selected for further reflection retrieval only if the crosses for both orthogonal lines fall within the chosen limits. This means that along both lines, the apparent velocities correspond to the velocities of the body waves and thus avoid the risk of also selecting arrivals such as the ones in Figure $4 \mathrm{a}$ and $4 \mathrm{~b}$. After this evaluation, 720 band-pass-filtered noise panels, which is approximately $3.5 \mathrm{~h}$ of passive data, are selected for the computation of the SI responses. The arrivals contained by the selected noise panels are characterized by slowness values ranging from -0.0005 to $+0.0005 \mathrm{~s} / \mathrm{m}$, which means apparent velocities of $2000 \mathrm{~m} / \mathrm{s}$ and higher. Most of the events have apparent velocities between 2200 and $5400 \mathrm{~m} / \mathrm{s}$.

From the slowness analysis, we now know the dominant slowness for each of the selected correlated panels. As explained above, due to the simple subsurface, we can now follow the method of Ruigrok et al. (2011) per selected correlated panel and use (concatenate) only specific parts of its retrieved positive and negative times. After that, the correlated panels are summed together to retrieve virtual common-source gathers.

In Figure 12a-12e, we display the virtual common-source gathers obtained for the master trace at geophones G1, G6, G12, G18, and G24 along the northeast line. The gathers are displayed after multiplying each sample by its corresponding time and then clipping. The time interval $0-0.6 \mathrm{~s}$ exhibits clear possible reflected waves characterized by strong amplitude. We can also see clear retrieved arrivals later than $1.2 \mathrm{~s}$. These arrivals are present on all retrieved common-source gathers.

The virtual common-source gathers obtained for master trace at geophones G1, G6, G12, G18, and G24 on the northwest line are also displayed in Figure $12 \mathrm{f}-12 \mathrm{j}$. Their arrival times are identical with those of the possible reflections seen on the gathers obtained along the northeast line. This coincidence in the arrival time is interpreted as confirmation that these arrivals can be considered as retrieved reflected waves. By comparing the virtual common-source gathers obtained on the northeast and northwest lines, we see that in the time interval $1-1.6 \mathrm{~s}$ the retrieved reflection arrivals are clearer along the northwest line.

Next, the retrieved virtual common-source gathers are processed using the same processing flow and parameters for the processing of the gathers obtained after the visual inspection of the noise. The resulting stacked section is shown in Figure 10b overlaid again on the stacked section from the active data.

Comparing the passive seismic sections displayed in Figure 10a and $10 \mathrm{~b}$, we see that the events in the latter are clearer and more continuous, for example, the geologic markers at $0.2,1.25,1.6$, and $1.9 \mathrm{~s}$. The geologic markers at 1.25 and $1.6 \mathrm{~s}$ are now clearly interpretable on the passive stacked section in Figure 10b, whereas in Figure 10a, they are not. We attribute the better results to the better suppression of surface waves in the retrieval process when the automatic slowness evaluation was applied to the noise. Comparing the active and passive sections displayed in Figure 10b, we can see that most of the reflectors from the active data can be followed to the passive section; for example, the packet of reflections seen in the two-way traveltime intervals $0.2-0.4 \mathrm{~s}$ and $1.25-2.0 \mathrm{~s}$.

The comparison between the active and the two passive sections in Figure 10 also reveals that there are differences as well. Concentrating on the passive section in Figure 10b, because its imaged results are clearer to interpret, we can see the difference in the retrieved amplitudes, but also in some retrieved arrivals that are not unambiguously interpretable in the active data. This might be explained in several ways.

One explanation is that it is possible to have different acousticimpedance contrasts on the geologic interfaces from the area where the passive data were recorded compared to lower contrasts in the area where the active data were recorded. This might explain, at least partly, the differences in amplitudes. On the other hand, previous geologic and geophysical studies in the area show that the geologic structures are represented by horizontal sedimentary layers with relatively small lateral variations in facies and lithology. This would make such changes in amplitudes and imaged arrivals not that likely.

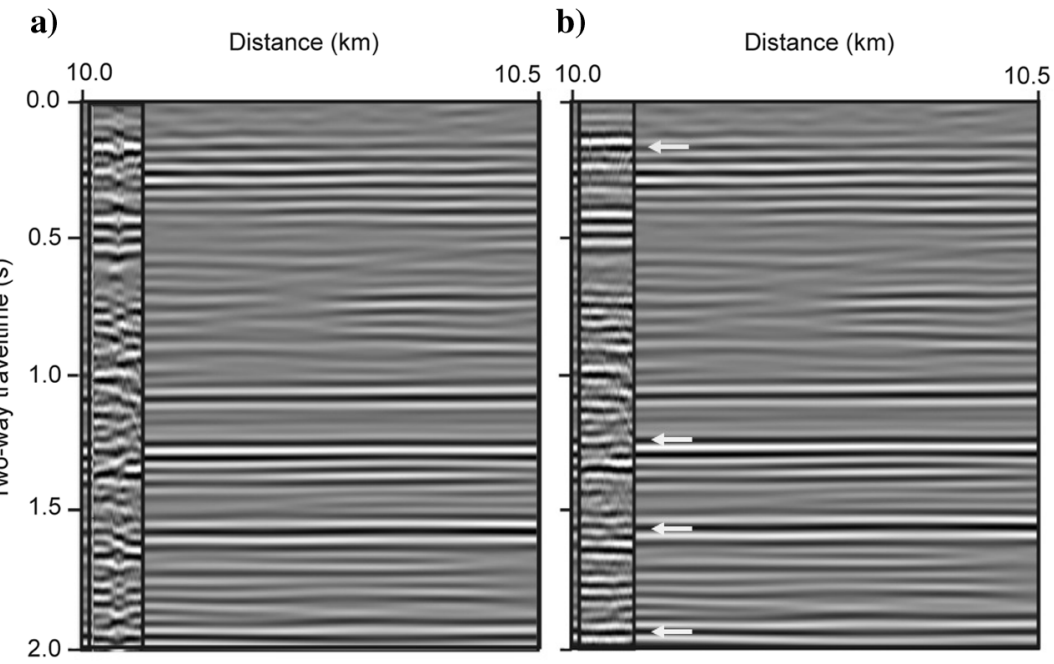

Figure 10. Stacked seismic section obtained from the passive survey along the northeast line (black line rectangle) overlaid on the stacked section from the active data. The passive results are obtained from noise panels selected after (a) visual inspection and (b) automatic slowness evaluation. White arrows are geologic markers.
Another explanation might be in the use in the retrieval process of the SI relation 1, which is approximate. According to the theory, to retrieve the correct Green's function between the geophones, one would need a monopole and a dipole source in the subsurface at the same source positions. This, of course, is unlikely to happen, so amplitude errors are expected. This might explain the difference in the amplitudes. Another possible result from the application of the approximate relation 1 is that ghost (nonphysical) arrivals are retrieved. The arrival at around $0.5 \mathrm{~s}$ in Figure $10 \mathrm{~b}$ might be a ghost. It might be retrieved, for example, due to the violation of the assumptions that the boundary sources in the subsurface effectively enclose the receivers and that the medium outside this source boundary is not causing backscattering that reaches the receivers. If any of these assumptions are violated, ghosts will be retrieved. When there is backscattering reaching the receivers, but the source distribution is sufficiently random in the vertical direction, the ghosts due to the 
backscattering will be suppressed (Draganov et al., 2006). Because we can see only a few possible prominent ghosts, the latter might be the case.

Both passive evaluation methods ensure that panels with bodywave noise are selected. The body waves might be reflected arrivals, but they might also be diving or refracted energy. After crosscorrelation, the diving and refracted energy will contribute to the retrieval only of diving and refracted waves, respectively. Due to the short length of our receiver lines, such arrivals will not be retrieved. In certain situations, however, the crosscorrelation of refracted energy might result in the retrieval of ghost arrivals as well. This might happen when the assumptions of smooth medium-parameter changes along the source boundary and/or energy reaching the receivers that does not leave the sources approximately perpendicular to their boundary (farfield assumption) are violated. In such cases, crosscorrelation of the first refracted arrivals would result in the retrieval of the so-called virtual refraction (Dong et al., 2006; Tatanova et al., 2008; Mikesell et al., 2009; Nichols and van Wijk, 2011). Such events pass through the virtual-source position at time $t=0 \mathrm{~s}$, exhibit inclination, and cannot be misinterpreted as possible retrieved reflections. On the other hand, crosscorrelation of the first refraction with its free-surface multiple would result in a virtual-refraction arrival that does not pass through $t=0 \mathrm{~s}$. Such a nonphysical arrival would be parallel to the earlier one and would appear later in time, delayed with the time of a critical reflection from the surface to the refraction layer. Such arrivals will show up at erroneous times in the retrieved stacked section when compared to the active section and might also be misinterpreted as possible retrieved reflectors.

\section{DISCUSSION}

In the previous section, we show results from the retrieval of reflections from ambient seismic noise. To bring forward the reflection arrivals above the otherwise dominant retrieved surface-wave arrivals, we use frequency band-pass filtering together with selection of which parts of the noise should be correlated. The selection is done using visual inspection and automatic slowness evaluation. Even though the results from both methods showed final results that allow interpretation of reflectors, the latter evaluation resulted in a better suppression of surface waves in the retrieval process and consequently in easier reflector interpretation. This can be seen, for example, comparing the retrieved common-source gathers for virtual sources at the position of geophone G24; see Figures 9e and 12e. In Figure 9e, retrieved surface-wave arrivals can still be seen distorting the retrieved nearly horizontal reflections down to $0.4 \mathrm{~s}$. The better suppression of surface waves using the automatic slowness evaluation comes from the fact that this method, in its current implementation, selects for further retrieval of reflections only those correlated panels, in which the dominant arrivals are caused by body-wave noise. The visual inspection, on the other hand, only ensured that the noise panels, which are selected for retrieval of reflections, contain identifiable body-wave noise.
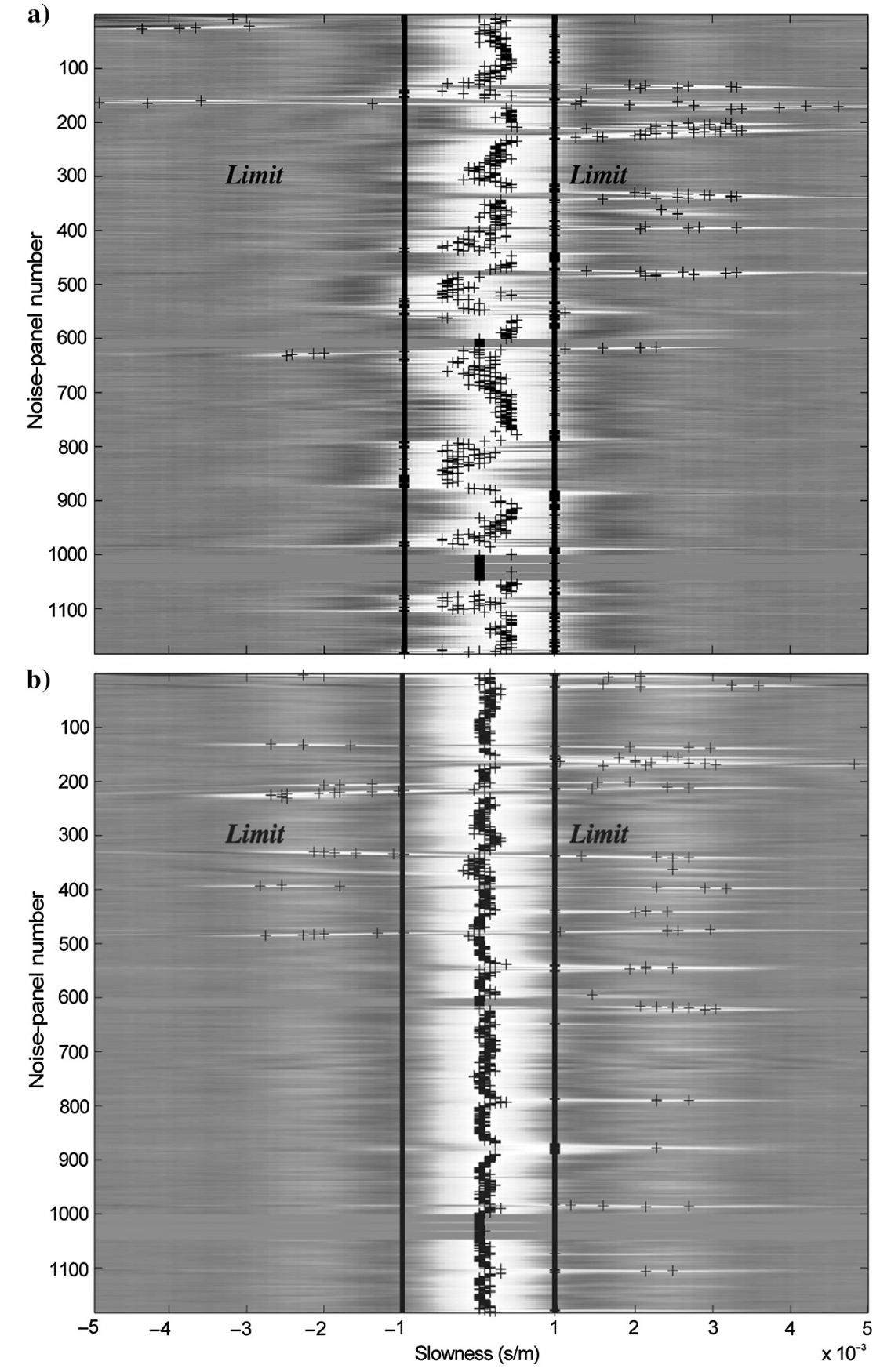

Figure 11. Slowness evaluation result for the (a) northeast and (b) northwest line. Crosses between the limits of -0.001 and $0.001 \mathrm{~s} / \mathrm{m}$ represent correlated panels that are dominated by body-wave noise in the selected frequency band, and crosses outside of the limits represent correlated panels dominated by surface-wave noise. 
Automatic slowness evaluation for selection of correlated panels dominated by body-wave noise should, though, be used carefully. The risk is that it can reject too many correlated panels. For example, if no band-pass filtering is applied to the recorded noise before

a)

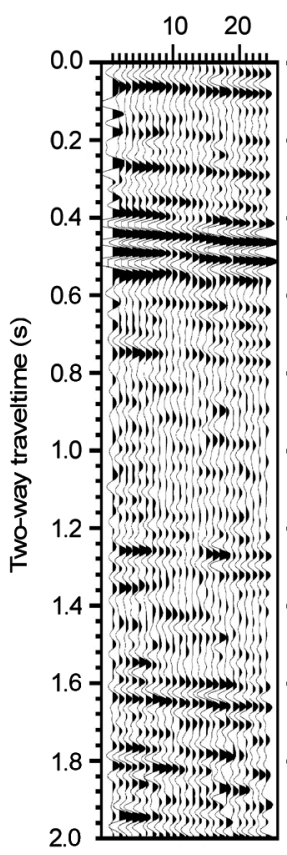

b)

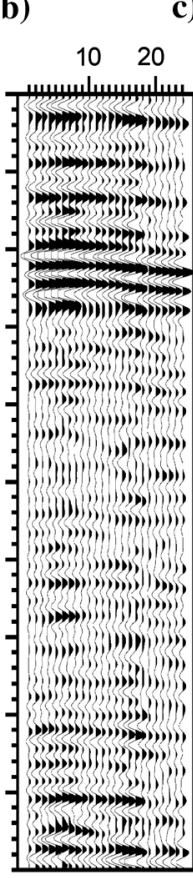

c)

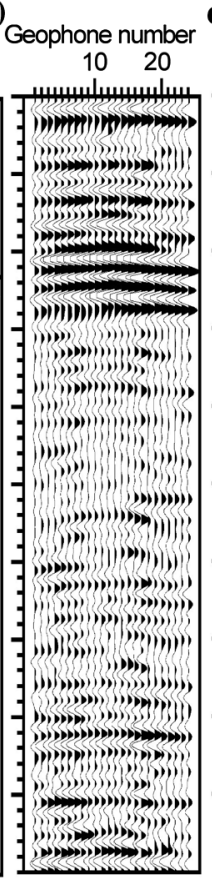

d)

$10 \quad 20$

e)

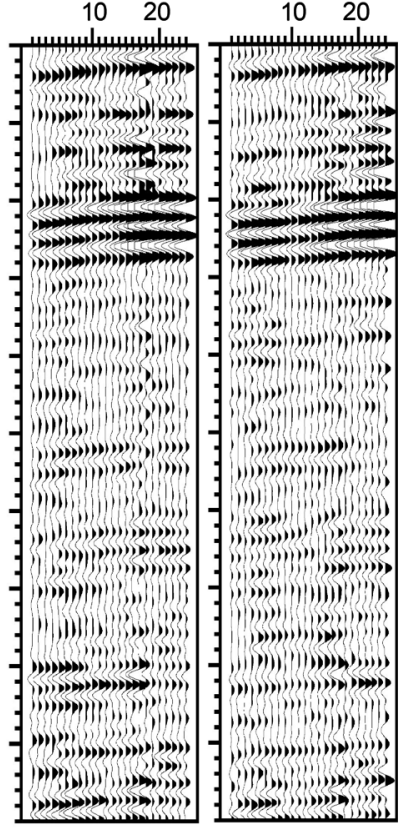

f)

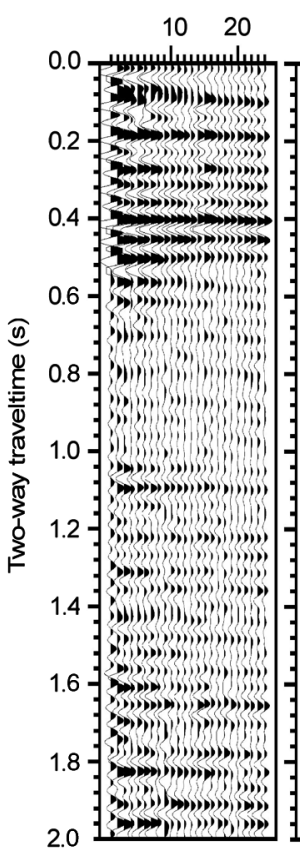

g)

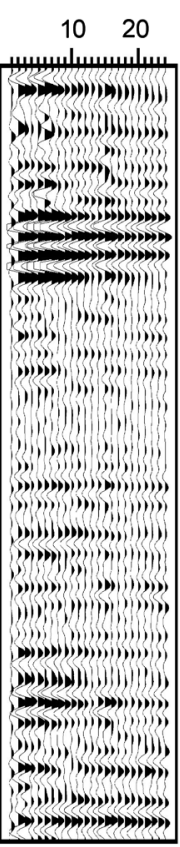

h)

Geophone number

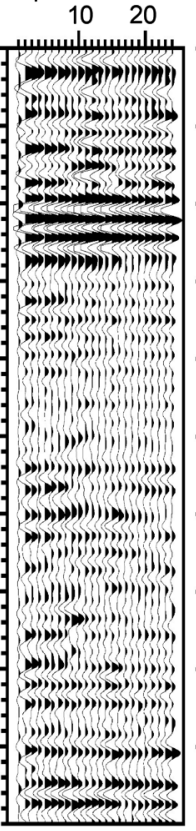

i)

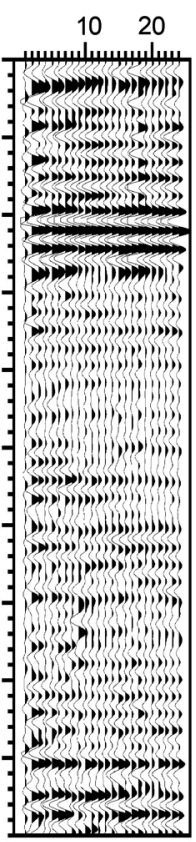

crosscorrelation, then nearly all of the data are rejected. When we use a band-pass filter between 11 and $23 \mathrm{~Hz}$, only approximately 350 correlated panels are selected for further reflection retrieval. After summation of the correlations, this number proved insufficient to improve the already obtained results. For this reason, the frequency spectra in Figure 3 were examined again, and we chose to use a band-pass filter between 15 and $23 \mathrm{~Hz}$ before the slowness evaluation. We apply the frequency filtering before slowness evaluation, but after the crosscorrelation. This slowness evaluation resulted in the selection of 720 panels for retrieval of reflections. The selected, but unfiltered, correlated panels were then summed together. Due to the desire to keep the frequency band of the retrieved results as broad as possible, the summed results were band-pass filtered between 11 and $23 \mathrm{~Hz}$. Even though this carries some risk of still retrieving some extra surface-wave energy, the broader frequency band helps the consequent interpretation of the retrieved results by suppressing apparent ringing at times when no strong arrivals are retrieved, like the time interval between 0.8 and 1.2 s. Changing the acceptance level during the slowness evaluation could further reduce the risk of rejecting too many correlated panels. For example, instead of accepting only correlated panels that are dominated by body-wave arrivals, we can select to accept panels in which the bodywave arrivals are comparable in strength to the surface-wave arrivals.

Another advantage of using the automatic slowness-evaluation method is that for each of the selected panels the dominant slowness along the two orthogonal directions is known. This gives the extra opportunity to determine the dominant illumination directions as detected at the passive array. Figure 13 shows an azimuthal plot of the illumination of the passive array obtained by converting the extracted slownesses to azimuth. A comparison of the main illumination direction with the distribution of the registered earthquakes in April 2009 from Figure 1 shows a good agreement between the two. Due to this, we speculate that the sources of the recorded body-wave noise might be low-magnitude earthquakes (lower than 0.5) related to the Campulung-Sinaia-Vrancea area. As we can see from the figure, the northeast line is illuminated predominantly from the northeast. This brings the risk of incomplete reflection retrieval from SI. The known illumination directions, together with the number of correlated panels contributing to each illumination direction, could be used to balance the retrieved results. This could be achieved by normalizing a correlated panel by the panel count for this illumination direction. Note that the selective combination of positive and negative
Figure 12. Retrieved common-source gathers obtained from noise panels selected for being dominated by body-wave noise after automatic slowness evaluation. The master trace is at geophones (a) G1, (b) G6, (c) G12, (d) G18, and (e) G24 along the northeast line and at geophones (f) G1, (g) G6, (h) G12, (i) G18, and (j) G24 along the northwest line. 


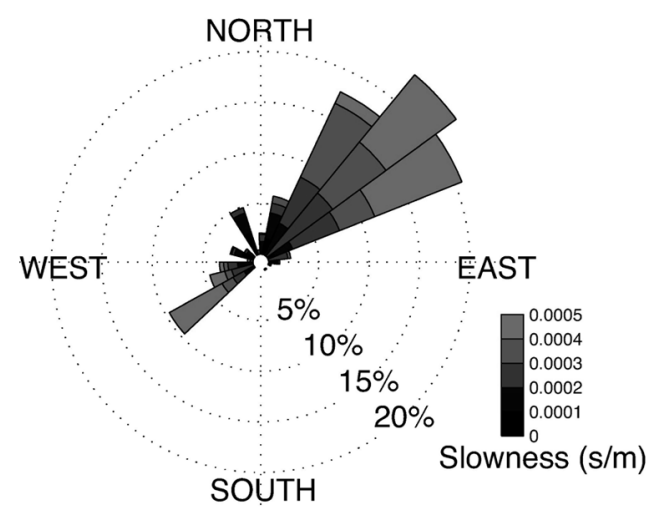

Figure 13. Azimuthal plot of the illumination of the passive array by body-wave noise. The azimuth was calculated from using the slowness values along both lines of the array. The slownesses are measured in the frequency band between 11 and $23 \mathrm{~Hz}$ from the crosscorrelated panels that were selected for retrieval of reflections.

times in the retrieval of the reflection gather in the case of automatic slowness evaluation, could be seen as "poor man's" balancing. For the northeast line, this results in obtaining the same illumination from the SW as for the northeast.

Another source of body-wave noise could be the earthquakes occurring in the vicinity of Romania. Six such earthquakes with epicenters in Turkey, Greece, and Italy were recorded at the Romanian seismological stations located in the Campulung-Sinaia-Vrancea area at the time interval of the ambient-noise measurements. Unfortunately, the arrivals related to these earthquakes are characterized by very low amplitudes and their identification on the seismological records is difficult. Looking in the ambient-noise data, the times at which these earthquake arrivals should be recorded are dominated by surface-wave noise, which makes the identification of these earthquakes impossible. Even if such earthquakes are identified, they would likely contribute to retrieval of body-wave reflections at frequencies lower than the ones we have retrieved.

Yet another source of body-waves noise could be traffic on the roads and the railway. Nakata et al. (2011) show that S-wave reflections could be retrieved from the traffic noise using SI by crosscoherence. The passive data were recorded in an area with heavy traffic. In our case, the traffic was very infrequent and the bodywave noise generated by it would be too weak to attempt retrieval with crosscoherence.

In the stacked section from the passive survey in Figure 10b, we can see events that do not coincide with reflectors in the active section. These events might be a result from possible refraction energy in the noise, as explained above. Another cause of such events in the retrieved results is the nonhomogeneous illumination of the passive array from the noise sources. As can be seen from Figure 13, there is a preferential illumination direction. During the summation process, this would lead to insufficient destructive interference and thus to the appearance of artifacts. Yet another potential cause for the ghost arrivals is the presence of intrinsic losses in the subsurface layers. Equation 1 was derived for a lossless medium; the application of this relation to measurements in a medium with dissipation would give rise to nonphysical arrivals in the retrieved result even if the illumination from the noise sources were homogeneous (e.g., Halliday and Curtis, 2009; Draganov et al., 2010b).

\section{CONCLUSIONS}

We applied SI to approximately $6 \mathrm{~h}$ of ambient noise recorded at Mizil, Romania, with the aim to retrieve reflected waves. The responses of SI were computed using noise panels with and without dominant surface waves as input data. In the case of noise panels dominated by surface waves, the SI results exhibited only retrieved surface waves. Array forming with five and 12 elements was tested to attenuate the surface waves in the recorded noise. The result with 12 elements was encouraging, but due to the short recording lines, we did not use this result for the retrieval of reflections.

We retrieved reflected waves after suppressing the surface-wave noise by frequency filtering followed by selection of recorded noise panels to be correlated. The selection was performed using two methods: visual inspection for selecting panels that are not dominated by surface-wave noise and automatic slowness evaluation for selecting crosscorrelated panels that are dominated by body-wave arrivals. We used the retrieved reflection gathers to obtain stacked subsurface images. Comparison of the two SI results with a stacked image from active seismic data showed that some retrieved events correspond to reflectors in the active data, including known geologic markers. Other retrieved events do not correspond to reflectors in the active data and might be retrieved nonphysical arrivals. The retrieved results had frequency content (imaging resolution) similar to the one from the active data. Better SI reflected waves were obtained after the automatic slowness evaluation. The evaluated slowness at the passive array suggests that the body-wave noise might be coming from the Campulung-Sinaia-Vrancea area.

\section{ACKNOWLEDGMENTS}

The passive seismic measurements were performed using equipment from Geometrics. C. Almagro Vidal is supported by the Netherlands Research Centre for Solid Earth Sciences. D. Draganov is supported by the Division for Earth and Life Sciences with financial aid from the Netherlands Organization for Scientific Research through Grant VIDI 864.11.009. We thank the associate editor, the reviewers G. Meles and N. Nakata, and two anonymous reviewers for their comments and suggestions that improved the quality of the paper.

\section{REFERENCES}

Almagro Vidal, C., J. van der Neut, D. Draganov, G. Drijkoningen, and K. Wapenaar, 2011b, Retrieval of reflections from ambient-noise field data using illumination diagnostics: 81st Annual International Meeting, SEG, Expanded Abstracts, 1613-1617.

Almagro Vidal, C., J. van der Neut, G. Drijkoningen, D. Draganov, and K Wapenaar, 2011a, Retrieval of reflections from ambient noise using the incident fields (point-spread function) as a diagnostic tool: 73rd Annual International Conference and Exhibition, EAGE, Extended Abstracts, P376.

Bakulin, A., and R. Calvert, 2004, Virtual source: New method for imaging and 4D below complex overburden: 74th Annual International Meeting, SEG, Expanded Abstracts, 2477-2480.

Bitri, A., G. Grandjean, and K. Samyn, 2011, Surface wave interferometry by cross-correlation and deconvolution: First Break, 29, 83-86, doi: 10 .3997/1365-2397.2011015.

Brenguier, F., N. M. Shapiro, M. Campillo, V. Ferrazzini, Z. Duputel, O. Coutant, and A. Nercessian, 2008, Towards forecasting volcanic eruptions using seismic noise: Nature Geoscience, 1, 126-130, doi: 10.1038/ ngeo104.

Claerbout, J., 1968, Synthesis of a layered medium from its acoustic transmission response: Geophysics, 33, 264-269, doi: 10.1190/1.1439927.

Curtis, A., and D. Halliday, 2010, Source-receiver wave field interferometry: Physical Review E, 81, 046601, doi: 10.1103/PhysRevE.81.046601. 
Curtis, A., H. Nicolson, D. Halliday, J. Trampert, and B. Baptie, 2009, Virtual seismometers in the subsurface of the Earth from seismic interferometry: Nature Geoscience, 2, 700-704, doi: 10.1038/ngeo615.

Dong, S., J. Sheng, and G. Schuster, 2006, Theory and practice of refraction interferometry: 76th Annual International Meeting, SEG, Expanded Abstracts, 3021-3025.

Draganov, D., X. Campman, J. Thorbecke, A. Verdel, and K. Wapenaar, 2009, Reflection images from ambient seismic noise: Geophysics, 74, no. 5, A63-A67, doi: 10.1190/1.3193529.

Draganov, D., X. Campman, J. Thorbecke, A. Verdel, and K. Wapenaar, 2010a, Event-driven seismic interferometry with ambient seismic noise: 72nd Annual International Conference and Exhibition, EAGE, Extended Abstracts, G013.

Draganov, D., X. Campman, J. Thorbecke, A. Verdel, and K. Wapenaar, 2013, Seismic exploration-scale velocities and structure from ambientseismic noise (>1 Hz): Journal of Geophysical Research, 118, 1-13, doi: 10.1029/2012JD018174.

Draganov, D., R. Ghose, E. Ruigrok, J. Thorbecke, and K. Wapenaar, 2010b, Seismic interferometry, intrinsic losses and Q-estimation: Geophysical Prospecting, 58, 361-373, doi: 10.1111/j.1365-2478.2009.00828.x.

Draganov, D., K. Wapenaar, W. Mulder, J. Singer, and A. Verdel, 2007, Retrieval of reflections from seismic background-noise measurements: Geophysical Research Letters, 34, L04305, doi: 10.1029/ 2006GL028735.

Draganov, D., K. Wapenaar, and J. Thorbecke, 2006, Seismic interferometry: Reconstructing the Earth's reflection response: Geophysics, 71, no. 4, SI61-SI70, doi: 10.1190/1.2209947.

Halliday, D., and A. Curtis, 2009, Seismic interferometry of scattered surface waves in attenuative media: Geophysical Journal International, 178, 419-446, doi: 10.1111/j.1365-246X.2009.04153.x.

Hoffe, B. H., G. F. Margrave, R. R. Stewart, D. S. Foltinek, H. C. Bland, and P. M. Manning, 2002, Analyzing the effectiveness of receiver arrays for multicomponent seismic exploration: Geophysics, 67, 1853-1868, doi: 10 $.1190 / 1.1527085$

Hohl, D., and A. Mateeva, 2006, Passive seismic reflectivity imaging with ocean-bottom cable data: 76th Annual International Meeting, SEG, Expanded Abstracts, 1560-1564.

King, S., and A. Curtis, 2011, Velocity analysis using both reflections and refractions in seismic interferometry: Geophysics, 76, no. 5, SA83-SA96, doi: 10.1190/geo2011-0008.1.

Mikesell, T. D., K. van Wijk, A. Calvert, and M. Haney, 2009, The virtual refraction: Useful spurious energy in seismic interferometry: Geophysics, 74, no. 3, A13-A17, doi: 10.1190/1.3095659.

Nakata, N., R. Snieder, T. Tsuji, K. Larner, and T. Matsuoka, 2011, Shearwave imaging from traffic noise using seismic interferometry by crosscoherence: Geophysics, 76, no. 6, SA97-SA106, doi: 10.1190/ geo2010-0188.1.

Nichols, J., and K. van Wijk, 2011, Application of the virtual refraction to near-surface characterization at the Boise Hydrogeophysical Research Site: Geophysical Prospecting, 58, 1011-1022, doi: 10.1111/j.13652478.2010.00881.x.

Oncescu, M. C., and K. P. Bonjer, 1997, A note on the depth recurrence and strain release of large Vrancea earthquakes: Tectonophysics, 272, 291302, doi: 10.1016/S0040-1951(96)00263-6.

Panea, I., and G. Drijkoningen, 2008, The spatial data-adaptive minimumvariance distortionless-response beamformer on seismic single-sensor data: Geophysics, 73, no. 5, Q29-Q42, doi: 10.1190/1.2969058.

Ravi Kumar, M., and M. G. Bostock, 2006, Transmission to reflection transformation of teleseismic wavefields: Journal of Geophysical Research, 111, B08306, doi: $10.1029 / 2005$ JB004104

Riley, D. C., and J. F. Claerbout, 1976, 2D multiple reflections: Geophysics, 41, 592-620, doi: 10.1190/1.1440638

Roux, P., K. G. Sabra, P. Gerstoft, W. A. Kuperman, and M. C. Fehler, 2005 P-waves from cross-correlation of seismic noise: Geophysical Research Letters, 32, L19303, doi: 10.1029/2005GL023803.

Ruigrok, E., X. Campman, and K. Wapenaar, 2011, Extraction of P-wave reflections from microseisms: Comptes Rendus Geoscience, 343, 512525, doi: 10.1016/j.crte.2011.02.006.

Sabra, K. G., P. Gerstoft, P. Roux, W. A. Kuperman, and M. C. Fehler, 2005 , Extracting time-domain Green's function estimates from ambient seismic noise: Geophysical Research Letters, 32, L03310, doi: 10.1029/ 2005GL023155.

Schuster, G. T., 2001, Theory of daylight/interferometric imaging: Tutorial 63rd Annual International Conference and Exhibition, EAGE, Extended Abstracts, A032.
Schuster, G. T. J. Yu, J. Sheng, and J. Rickett, 2004, Interferometric/ daylight seismic imaging: Geophysical Journal International, 157, 838-852, doi: 10.1111/j.1365-246X.2004.02251.x.

Sens-Schönfelder, C., and U. Wegler, 2006, Passive image interferometry and seasonal variations of seismic velocities at Merapi Volcano, Indonesia: Geophysical Research Letters, 33, L21302, doi: 10.1029/ 2006 GL027797.

Shapiro, N. M., and M. Campillo, 2004, Emergence of broadband Rayleigh waves from correlations of the ambient seismic noise: Geophysical Research Letters, 31, L07614, doi: 10.1029/2004GL019491.

Shapiro, N. M., M. Campillo, L. Stehly, and M. H. Ritzwoller, 2005 , High-resolution surface-wave tomography from ambient seismic noise: Science, 307, 1615-1618, doi: 10.1126/science.1108339.

Slob, E., D. Draganov, and K. Wapenaar, 2007, Interferometric electromagnetic Green's functions representations using propagation invariants: Geophysical Journal International, 169, 60-80, doi: 10.1111/j.1365246X.2006.03296.x.

Snieder, R., 2004, Extracting the Green's function from the correlation of coda waves: A derivation based on stationary phase: Physical Review E, 69. 046610, doi: 10.1103/PhysRevE.69.046610.

Snieder, R., 2007, Extracting the Green's function of attenuating heterogeneous acoustic media from uncorrelated waves: Journal of the Acoustical Society of America, 121, 2637-2643, doi: 10.1121/1.2713673.

Tatanova, M., A. Bakulin, K. Mehta, V. Korneev, and B. Kashtan, 2008 Reconstructing head waves with virtual source method: 78th Annual International Meeting, SEG, Expanded Abstracts, 183-187.

Tonegawa, T., K. Nishida, T. Watanabe, and K. Shiomi, 2009, Seismic interferometry of teleseismic S-wave coda for retrieval of body waves: An application to the Philippine Sea slab underneath the Japanese Islands: Geophysical Journal International, 178, 1574-1586, doi: 10.1111/j .1365-246X.2009.04249.x.

van der Neut, J., 2013, Downhole interferometric illumination diagnosis and balancing: Geophysical Prospecting, 61, 352-367, doi: 10.1111/j.13652478.2012.01122.x.

van Manen, D.-J., J. O. A. Robertsson, and A. Curtis, 2005, Modeling of wave propagation in inhomogeneous media: Physical Review Letters, 94 164301, doi: 10.1103/PhysRevLett.94.164301.

Vasconcelos, I., and R. Snieder, 2008a, Interferometry by deconvolution, Part 1 - Theory for acoustic waves and numerical examples: Geophysics, 73, no. 3, S115-S128, doi: 10.1190/1.2904554.

Vasconcelos, I., and R. Snieder, 2008b, Interferometry by deconvolution: Part 2 - Theory for elastic waves and application to drill-bit seismic imaging: Geophysics, 73, no. 3, S129-S141, doi: 10.1190/1.2904985.

Wapenaar, C. P. A., J. W. Thorbecke, D. Draganov, and J. T. Fokkema, 2002 Theory of acoustic daylight imaging revisited: $72 n d$ Annual International Meeting, SEG, Expanded Abstracts, 2269-2272.

Wapenaar, K., and J. Fokkema, 2006, Green's function representations for seismic interferometry: Geophysics, 71, no. 4, SI33-SI46, doi: 10.1190/1 .2213955 .

Wapenaar, K., E. Ruigrok, J. van der Neut, and D. Draganov, 2011a, Improved surface-wave retrieval from ambient seismic noise by multidimensional deconvolution: Geophysical Research Letters, 38, L01313, doi: 10 $.1029 / 2010$ GL045523.

Wapenaar, K., J. van der Neut, and E. Ruigrok, 2008, Passive seismic interferometry by multi-dimensional deconvolution: Geophysics, 73, no. 6 , A51-A56, doi: 10.1190/1.2976118.

Wapenaar, K., J. van der Neut, E. Ruigrok, D. Draganov, J. Hunziker, E. Slob, J. Thorbecke, and R. Snieder, $2011 \mathrm{~b}$, Seismic interferometry by crosscorrelation and by multidimensional deconvolution: A systematic comparison: Geophysical Journal International, 185, 1335-1364, doi: :10.1111/j.1365-246X.2011.05007.x.

Xu, Z., C. Juhlin, O. Gudmunsson, F. Zhang, C. Yang, A. Kashubin, and S. Luth, 2012, Reconstruction of subsurface structure from ambient seismic noise: An example from Ketzin, Germany: Geophysical Journal International, 189, 1085-1102, doi: 10.1111/j.1365-246X.2012.05411.x.

Yao, H. R. D. van der Hilst, and M. V. de Hoop, 2006, Surface-wave array tomography in the SE Tibet from ambient seismic noise and two-station analysis: I - Phase velocity maps: Geophysical Journal International, 166, 732-744, doi: 10.1111/j.1365-246X.2006.03028.x.

Yilmaz, O. 1999, Seismic data processing, 9th ed. SEG.

Zhan, Z., N. Sidao, D. V. Helmberger, and R. W. Clayton, 2010, Retrieval of Moho-reflected shear wave arrivals from ambient seismic noise: Geophysical Journal International, 182, 408-420, doi: 10.1111/j.1365246X.2010.04625.x. 